Institute of Information Technology ANAS, Baku, Azerbaijan rasimmahmudov@gmail.com

\title{
ABOUT FEATURES OF SOCIAL MEDIA
}

The article examines the communications, information, value generation, organization, and entertainment functions of social media. The features of these functions are commented in the article. Respective features of traditional and social media are compared.

Keywords: social media, communication function, information function, value creation function, organizational function, entertainment function.

\section{Introduction}

The Internet and social media, its integral part, is attributed to social communication institution - communication tool and information exchange. The performance of social media, as a social institute, is determined by the requirements and interests of various social entities. In sociology, by investigating the Internet and social media, the impact of this global phenomenon on human life, the evaluation of alteration of reflections regarding the objective social needs of an individual, separate groups and the society as a whole are studied.

Not only the fact of behavior, but also the motives behind this behavior are revealed in social studies. Sociologists attempt to study the factors (causes) facilitating the behavior and the outcomes of the use of corresponding communication system as well. They endeavor to forecast by evaluating (measuring), describing and interpreting the events occurring in this sphere and determining existing legal framework.

Empirical investigations in social media sphere is mainly beneficial for the employees of this sphere. Therefore, such investigations are important for decision-making on new media-projects, editing the content and target audience. Moreover, sociologists and analysts analyzing social processes (Internet-media as an integral part of it), media experts analyzing various mass media activities and the consumer markets, marketing specialists, advertisers, and others are social research subjects also for experts of this sphere [1].

The outcomes of standard and regular investigations (mediametry), targeting the mass customers and potential users, are directed towards the satisfaction of market needs. Specific or exclusive studies are usually carried out by orders and by state or private grants. The results of mediametry is also important for socio-political analysts.

Internet as a basis of social media is also an environment of operation of various communications. An American political scientist Harold Lasswell reckoned that every social communication fulfills the main three functions: executing the control on environment, acting as a communication tool among social entities and facilitating the culture (knowledge, values, norms, traditions) transmission to future generations $[2,3]$.

Universal communication functions can also be attributed to the Internet. Historical development of mass media has caused the emergence of several specific functions in this sphere. Those functions have been carried out by the impact of enhancement of technological facilities of socio-political and social economic situations, information generation and dissemination in specific countries. For instance, the function of mass media, as a social inclusion, has started to be perceived since the midst of the past century. With the emergence of the Internet, the realization capacity of this function has significantly increased. However, this function is being realized at very low levels in such environment. In this regard, M. Castells mentions that a good or bad character of technology depends on how those technologies are applied by us [4].

Social media possesses traditional functions of mass media, and generates new functions pertaining to this technological environment as well. The study of characteristics of those functions has a socio-political and socio-cultural importance alongside the scientific relevance. 


\section{Communication function of social media}

One of the most important functions carried out by social media is communication function. As known, communication tools are created in order to provide people with sociability tools. Mass communications serve to the creation of communications among mass entities. The communication is constructed via internet-media such that professional information producer and mass entities - the mass of anonymous users, the number of each is inestimable, stand on either sides.

Internet-technologies globalize the communications. The dependence of communications on territorial factors evoperates emerges in such curcimstance. Internet-media, benefiting from such opportunities, shapes a new society based on unified information basis and the code of conduct.

The general vector of the development of modern information transmission tools is based not on vertical (domination of a specific communication party upon other) but on heterarchical (horizontal, equal) principle. In other words, the transition from one-sided communication model to two-sided communication model takes place.

Social media is not excluded from these changes in the communication sphere. Not only a journalist, but also the audience participates in information generation and exchange. However, the use of interactive opportunities in this sphere does not allow the distribution of responsibility division. Three aspects of network interactivity are distinguished [5]:

- "People and documents" (the opportunity to shape and carry out information request for a user);

- "People and technologies" (the convenience and the adaptability potential of information technologies for users);

- "People and people" (social communication technologies, the appropriateness and convenience of those for mutual communication).

While analyzing the communication function of the social media, essence of the notions such as "interactivity" and "feedback" must be explained. Firstly, it is to be mentioned that the first notion is wider than the latter. Feedback - is a reaction, a response of an entity to information influence. From this point of view, social media makes use of "forum" function in greater sense. Visitor indicators also act as feedback: it provides statistical information regarding the degree of interest of user in a web-site and its content. Interactivity reflects wider opportunities: it contemplates user control on content (request, evaluation), the participation in content creation, content discussion, conference with other users, and etc. Some of these opportunities used to pertain to media as well. But, the opportunity of horizontal communication among users is only present in social media. It is also impossible to maintain communication operativeness in traditional media.

H. Lasswell distinguishes three levels of interactivity [2]:

- Mutual, but non-interactive communication (for instance: phone calls, mails)

- Reactive and quasi-reactive communication, i.e. one party receives information about the reaction of other party (feedback)

- Fully interactive communication, i.e. all communication entities participate in information exchange based on equal opportunities.

At present, the audience of social media is able to use sufficiently enhanced communication tools: discussion of a particular article or program, filling forms as a response or answers to editorial questions, the participation in rating evaluation of materials, sending questions to editorial office, a particular journalist or speaker, general evaluation of performance of a channel or web-site, the discussion of social problems and political issues, the dispatch of materials for publishing and etc.

Consequently, unlike traditional media, social media possesses more specific communicative functions. Internet creates enhanced opportunities for maintenance of mutual communication and interactivity. 
The information contained in social media pages is accessible by everyone. Only limitations related to payment can occur. The problem of maintenance of equal accessibility to resources is one of the major issues related to sociological studies and practical development of the Internet. Optimists reckon that social media created feasible conditions for maintenance of equal opportunities for groups previously deprived of these opportunities with other groups. However, in presence of digital inequality, the process can develop in reverse direction, i.e. the social gap can widen.

Information in social media possesses several following features as in traditional media [5]:

- operativeness;

- Illumination of social life;

- Universality and versatility of subject matter (even being a specialized media tool);

- Genre-type variety of materials.

The absence of specification of legal responsibility relieves the sense of responsibility for materials posted in social media. This leads to the increase of misinformation, unclarified and unreliable information.

\section{Information function of social media}

Multimedia feature of social media creates new opportunities for realization of information function. It is well known from the practice of traditional media that different marking and influence tools or the variety of information introduction-reception channels ease the information and knowledge assimilation. Hypertext feature of social media significantly enhances the opportunities of information function.

Therefore, it is to be mentioned that multimedia as a new and universal sphere of social media makes information more multi-colored and interesting in terms of type, form and genre. Internet possessing multimedia and hypertext features transforms the information into more enriched, comprehensive and solid form [1].

\section{Value creation function of social media}

It must be noted that family and schools shape the first socialization. Thus, an individual familiarizes himself with knowledge, norms, values, practice and traditions collected in society and surrounding social microenvironment. In a modern society, this function substantially lies on media. Media serves to value creation and conservation.

Social media takes the freedom of information exchange to the next quality level. Global network also aggrandizes the issue of social identity (belonging of individual to particular social groups). If traditional communications (mail, book, telephone and etc.) have eliminated spacetime obstacles, Internet has gone further - it eliminated social identity. It means that the Internet has become a space of rapidly alternating wide-spectrum virtual societies. As a result, global valuenorm basis started to be formed [1].

In a modern independent journalism, a fact is accurately distinguished from an opinion. Therefore, an opportunity is created for a reader, listener and spectator to draw one's own conclusions. The realization of this function in internet-journalism is dependent upon the journalist culture, admission of responsibility for editorial policy, possession of skills of various genres and methods of expression in relation to an object of illustration. It also depends upon user culture the ability to use the Internet opportunities for shaping personal position. It is known from the investigation of traditional media that some users try to use various, also alternative information in order to shape his views, whereas other users only wish to benefit from suggested position.

Value creation function is focused on the information sharing and attraction of attention of audience towards events and problems, and shaping the agenda. On the Internet, the process of division and split of audience to several groups is observed. In general, social media is able to carry out this function pertaining to traditional media. Moreover, the features of Internet such as 
operativeness, interactivity and hypertexting ascends this function onto a new qualitative level. The expansion of division process of the audience of offline and online channels, asyncronization of information perception by audience disturbs communion of information-value platform and "agenda" [6].

\section{Organizational function of social media}

Media participates in operation of real social groups and institutions - town residents, employees of a particular field or members of public union, and in realization of their functions. This is called organizational function [1].

It is affirmed that mass media is a specific society comprising the activities on satisfaction of information requests via a particular channel. Based on long-term practice, the proximity of views is strengthened, base of general information and values is established, the processes of exchanging opinions, related to socio-political issues, are developing. According to several sociologists, new groups emerge in general communication base related to general cultural interests, similar problems and needs to express own views [7].

Therefore, the Internet creates a feasible environment for formation of social unity, solidarity and identity. Sociology and social sciences affirm that the audience of each media tool constitutes a specific society. Common views, information reserves and values of individuals, united to satisfy information needs from the same channel, are created. Internet breathes a new life into this process. It becomes an easy and rapid process to unite people around particular interests via social networks, forums, chats [8]. According to M. Castells, Internet upgrades old communication tools of an individual and society. If previously these methods were based on areal factor, now it is based on "network individuality". Multiple links are referred to "weak links" on the Internet; however, they are very important and can even maintain family societies [9].

In the first years of mass penetration of the Internet, activists of various public unions reckoned that the global network cannot supersede the real human communication. However, subsequent practice demonstrated that new technological communication tools among individuals have own benefits. It is revealed that these communication tools are very suitable for social solidarity and elimination of digital inequilaty. Therefore, it can be stated that social media cannot supplant real human communication, but it can enrich such communication channels.

\section{Social inclusion function of social media}

Another function of media created by the Internet is a social inclusion function. The notion of the "social inclusion" has emerged in the 30's of the past century. By "social inclusion", various methods of citizen activity in discussion and solution of important issues are understood [1].

Communicative participation or inclusion in social communication is deemed more accessible form of social inclusion for population stratums. The necessity, possibility and effectiveness of this participation via traditional media has been discussed for several decades.

If in totalitarian and authoritarian regimes a journalist is a guarantor information interests of a government and protector of information gate, in open societies a journalist strives to open those doors wider.

In modern society, media acts as an important integral part of public sphere. Here, mutual relations of main social entities - society, government and business is transformed into discussion and judgement with journalist participation. Some sociologists contend that the rapid development of information technologies creates new expectations in this sphere. Hence, it enables the gap elimination, and establishment of communication between citizens and the government. In other words, this new situation acts as a stage of realization of electronic democracy or direct democracy.

Social media is an environment, in which not only the space limitations, but also the obstacles in realization of separate spheres of human activity - work and everyday life, hobby and civil status, and the differences between education and entertainment are eliminated. Information 
maintenance of these spheres and tools for participation in them located in a unified media environment, and the distances among different spheres are almost absent.

Before the Internet, some of these facilities were provided by traditional media, including television. However, interactive and online opportunities of Internet sustain its supremacy against traditional media.

\section{Entertainment function of social media}

The function of entertainment captures a significant place in operation of social media. The rapid increase of Internet users, widening of social-demographical composition and geography, elimination of differences in Internet access among population groups have transformed the social media into the most interesting entertainment place. Nevertheless, experts held a different opinion 15 years ago in this regard. M.Castells excepted the opinion that Internet would be able to compete with traditional entertainment tools and places. He considered that Internet is not an entertainment, but a communication tool [8].

Traditional and social media are capable to support each other in a more effective conduction of social functions. Modern tendencies indicate that centralization, convergence and integration in a single center (holding, editorial office, publishing house and etc.) of all types of media tools are taking place. Electronic versions of almost all traditional media tools are present. In the days of the Soviet era, different incentives have been suggested regarding the necessity of mutual functioning of different media instruments. At that time, different ideas such as "The Union of microphone, pen and video camera" (V.Khelmendik), "Solo parts in ensemble" (V.Sesyunin) had been discussed [5]. The essence of these incentives suggested the necessity of comprising specific facilities of each media tool and acting on a unified platform in order to effectively impact the audience.

The most prominent social outcomes of joint, mutual functioning of traditional and social media are the enhancement of information volume and variety, and the increase of operativeness, in which the main role is attributed to the Internet.

On the one hand social media accelerates the tendency of audience fragmentation and performs in a reverse direction, on the other hand it crystalizes same brand in different channels. For instance, if the audience of a traditional publication - a newspaper, is mainly constituted of middle-aged and old generation, and the electronic version of that publication attracts teenagers and young people.

By using information update tools of various speed, the integration of facilities of offline and online versions is very effective for satisfaction of different audience groups. In traditional media, especially in radio and television, different contents are updated or repeated several times during a day. For instance, news program is broadcasted several times during a day, weekly information-analytical programs, monthly thematic programs are prepared. In social media, the programs, updated with different periodicity, can be placed on the same web site, i.e., different user groups, at the same time, can select the contents they are interested in. Hence, audience segmentations (fragmentation) and diversification of information materials (the increase in variety) take place. Alongside, coordination facilities of paper and electronic media is being widened, the processes of labor division and specialization in journalism are accelerated due to the audience with different needs (the increase in information needs of different purposes) [1].

In the environment of mutual functioning, the greatest cooperation opportunities are created from the point of view of mutual communication of journalists with the audience and within the audience. The audience using the Internet can influence the content and editorial plans, which is called "social control over media". 


\section{Conclusion}

Distinctive features of social media against traditional media are clearly reflected in functions carried out by social media. Traditional functions of media have acquired enhanced opportunities in quantitative and qualitative terms in the social media era. Moreover, social media carries out news and unique functions. which are beyond the capacity of traditional media. All these facilities, based on technological foundations, are directed towards the comprehensive satisfaction of information, communication and other civil needs of people. The emergence of more unique functions of social media is expected within dynamic development process of social media technologies. All these necessitate non-stop expansion of social media audience and acquisition of the competitive dominance over the traditional media.

\section{References}

1. Alguliyev R.M., Mahmudov R.Sh. The emergence and development of Internet-media: main trends and features. Express-information. Information society series. Baku, "Information Technologies" publishing house, 2012, $58 \mathrm{p}$.

2. Lasswell H. Structure and Function of Communication in Society / Bryson J.(ed.) The Communication of Ideas, N.Y., The Free Press, 1948, 315 p.

3. Casmir F.L. Building Communication Theories: A Socio/cultural Approach, Psychology Press, 1994, $321 \mathrm{p}$.

4. Castells M. Information age: Economy, Society and Culture. Trans. in Russian by State Univ. of Higher school of Economics. Moscow, 2000, 606 pp.

5. Gnatyuk O.L. Foundations of communication theory. Moscow, "Knorus", 2010, 256 pp.

6. Fomicheva I.D. Mass media sociology: manual for graduate students. Moscow: "Aspect Press", 2007, 335 p.

7. Reitz A. Social Media's Function in Organizations: A Functional Analysis Approach // Global Media Journal - Canadian Edition, 2012, Volume 5, Issue 2, pp. 41-56

8. Reinhold G. Smart mobs: The next social revolution, Moscow, "FAIR-PRESS", 2006, 416 p.

9. Castells M. The Internet Galaxy: Reflections on the Internet, Business and Society /Trans. from English, Ekaterinburg, "U-Faktoria", 2004, 328 p. 